\title{
Speed of Online and Print Peer-Reviewed Ophthalmology Publications and Correlation to Journal Bibliometric Measures
}

\author{
Ronaldo Nuesi, BS ${ }^{1,2}$ John Y. Lee, BS ${ }^{1}$ Ajay E. Kuriyan, MD $^{3}$ Jayanth Sridhar, MD ${ }^{1}$ \\ ${ }^{1}$ Department of Ophthalmology, Bascom Palmer Eye Institute, \\ University of Miami Miller School of Medicine, Miami, Florida \\ 2 Herbert Wertheim College of Medicine, Florida International \\ University, Miami, Florida \\ ${ }^{3}$ Department of Ophthalmology, Sidney Kimmel Medical College of \\ Thomas Jefferson University, Retina Service/Mid Atlantic Retina, \\ Wills Eye Hospital, Philadelphia, Pennsylvania \\ Address for correspondence Jayanth Sridhar, MD, 900 Northwest \\ 17th Street, Miami, FL 33136 (e-mail: jsridhar1@med.miami.edu). \\ J Acad Ophthalmol 2020;12:e284-e291.
}

\begin{abstract}
Keywords

- publication speed

- ophthalmology

- bibliometric

- journal impact factor

- Eigenfactor score

- Citescore
\end{abstract}

Objective This study aimed to explore the relationship between publishing speeds and peer-reviewed journal bibliometric measures in ophthalmology.

Methods Journal Citation Reports and Scopus Database were accessed for identification of journal bibliometric measures in ophthalmology. Twelve randomly selected articles from 2018 for all identified journals were studied. All outcome measures were extracted from the full text of articles and correlated with journal bibliometric measures. Statistical analysis was performed on measured parameters in comparison to a previous study.

Main Outcomes and Measures Journal impact factor, Eigenfactor score, and CiteScore were correlated with time from submission or acceptance of manuscripts to online and print publication. The correlation between study design and publishing speeds was also assessed.

Results A total of 55 journals were included for a total of 657 articles. Online publications were significantly faster than print publications for almost every journal $(p<0.001)$. Laboratory experimental studies had significantly shorter times from submission to online publication $(p=0.002)$ and acceptance to online publication $(p<0.001)$ compared with observational and interventional studies. Journal impact factor was positively correlated to publishing speed from acceptance to online publication ( $p=0.034)$. CiteScore was positively correlated to speed from submission to print publication $(p=0.04)$, acceptance to print publication $(p=0.013)$, and acceptance to online publication $(p=0.003)$. Eigenfactor score was not statistically significant when correlated with any outcome measures.

Conclusion Online publication has increased speed of dissemination of knowledge in the ophthalmology literature. Despite reporting higher numbers of submissions every year, ophthalmology journals with higher bibliometric measures of impact tend to publish peer-reviewed articles faster than journals with lower impact scores. Study design of an article may affect its speed to publication. received

August 16, 2020

accepted after revision

September 14, 2020
DOI https://doi.org/

10.1055/s-0040-1721070.

ISSN 2475-4757.
Copyright $\odot 2020$ by Thieme Medical

Publishers, Inc., 333 Seventh Avenue, New York, NY 10001, USA. Tel: $+1(212) 760-0888$.
License terms

(®) (1) $\Theta \circledast$ 
With an exponentially increasing amount of available peerreviewed literature, publishing in a highly regarded journal has become even more necessary for increased visibility of one's research and may be a factor for academic promotion. ${ }^{1}$ In recent years, the majority of medical journals have made the transition to online publication of peer-reviewed manuscripts ahead of printed format. As a result, the relative ease of access to published research in a theoretically timely fashion is unparalleled; however, high-impact journals report increasing numbers of submissions every year, and this could theoretically lead to increased times to publication because of the arduous process that must go into peer review of each article. Slower publication speeds may result in delay in dissemination of crucial information, and as the novel corona virus disease 2019 (COVID-19) viral pandemic has illustrated, expedient availability of new research is critical to institute new diagnostic and treatment measures.

There is little research to tie speed to publication to the "impact" of a journal in the ophthalmology literature. In 2013, Chen et al evaluated the time from submission to printed publication, time from acceptance to printed publication, and time from submission to acceptance for articles published in 2010 to determine if these parameters correlated to the journal impact factor (JIF) in ophthalmology journals and found no statistically significant correlations between journal impact factors and these parameters; however, they reported that journals with advance online publication had significantly higher JIFs when compared with print only journals. ${ }^{2}$ For context, only 26 journals in 2010 had online advance publications, ${ }^{2}$ and it is unknown whether greater speed of online publication is correlated with JIF.

Today, every ophthalmology journal offers online publication, and the current study evaluates how time from submission to online publication, from acceptance to online publication, and from online publication to print publication are correlated with JIF. In addition, this study examines two other bibliometric measures, CiteScore (CS), and Eigenfactor score (ES), which have become more prominent in recent literature, and assesses whether the study design influenced speed of publication. ${ }^{3-8}$

\section{Methods}

The Journal Citation Reports for 2018 was accessed and filtered for the category of ophthalmology (available at: $h t t p: / / w w w$. webofknowledge.com/JCR; May 29, 2020). Sixty journals were present in the report. Review journals were excluded to diminish the effects of distinct publication timelines for invited review articles. A list of bibliometric measures for all identified ophthalmology journals was obtained. JIF and ES were selected for evaluation. Additionally, a list of the CS for these 55 journals was obtained through Scopus (available at: https://www.scopus.com/sources.uri; May 29, 2020). When sufficient articles were available, 12 articles were randomly selected from the 2018 volumes of each journal. One article from each issue was chosen using a random number generator for journals with 12 issues. In journals with six issues, two articles were selected at random from each issue. In journals that did not have 6 or 12 issues, one article was randomly chosen from each issue and then a random number generator was used to select issues from which to choose the remainder of articles. Supplementary issues were excluded. All articles were selected from the original investigations section of an issue. Review articles were excluded for the same reason as outlined above for review journals.

The submission, revision, acceptance, online publication, and printed dates were recorded from the full text of each article if available. All randomly selected articles were included despite missing information unless submission date was not provided and the journal had other articles that provided submission dates. In these cases, another article from the same issue was chosen at random. If the journal did not report submission dates for any articles, all initial randomly selected articles were included. The article study type (basic or clinical), study design (observational, interventional, or laboratory experiments), and study results (positive or negative) were recorded and totaled up for stratification. Kruskal-Wallis test was performed to assess for parameter differences between the three study designs. Mann-Whitney $U$-test further analyzed grouped differences in study designs identified by Kruskal-Wallis test.

The time lag between submission and revision, acceptance, online publication, and printed publication was calculated (in days) along with the period from acceptance to online publication and printed publication. The median for each parameter, as well as the interquartile range (IQR), was calculated for each journal. Spearman's correlations between each parameter and the JIF, ES, and CS were analyzed. Spearman's correlation between JIF, ES, and CS was analyzed to confirm equal measurements. Wilcoxon's signed-rank test was used to compare online publication times and print publication times for each journal. Wilcoxon's test was also performed to analyze the data as it compared with the study by Chen et al. ${ }^{2}$ Spearman's correlations in Chen et al were also compared with those included in this study. All statistical analyses were performed with SPSS software Version 26.0 (SPSS, Inc., Chicago, IL).

\section{Results}

\section{Journal Characteristics}

After exclusion of all review journals, 55 journals were analyzed (-Table 1). Every journal in the study had online publications of their articles available. Five journals (9\%) did not report submission dates for any article and were excluded from final analysis of all time points except online to print publication time. Thirty-six journals (65.4\%) did not report revision dates. Six journals (10.9\%) did not report acceptance dates. Nine journals (16\%) did not report their online publication dates; however, online ahead publications were present for all nine. Nine journals (16\%) did not report print publication dates of which four were online only journals.

\section{Article Characteristics}

A total of 657 articles from 55 journals were included for analysis; one journal had only nine available original research articles for 2018 (Visual Neuroscience). For all randomly 


\begin{tabular}{|c|c|c|c|c|c|c|c|c|c|c|c|c|c|c|c|c|c|c|c|c|c|c|c|c|c|c|c|c|c|c|}
\hline 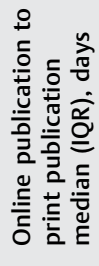 & 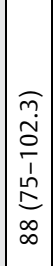 & 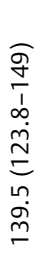 & 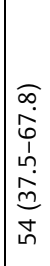 & | & $\frac{1}{z}$ & 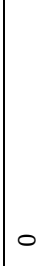 & 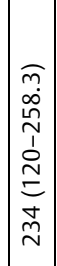 & 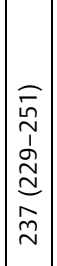 & 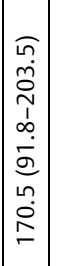 & 0 & $\delta$ & 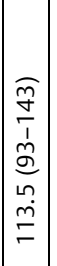 & $\frac{\mathbb{z}}{z}$ & 0 & $\frac{\mathbb{s}}{z}$ & $\frac{\mathbb{Z}}{z}$ & $\frac{\mathbb{s}}{z}$ & 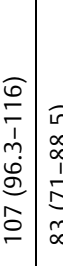 & 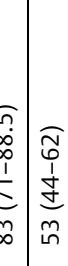 & $\frac{s}{z}$ & $\frac{s}{z}$ & 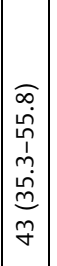 & $\frac{5}{z}$ & $\frac{s}{2}$ & 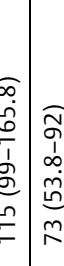 & 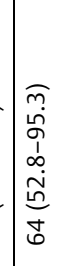 & 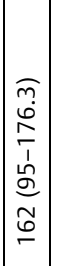 & 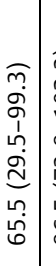 & 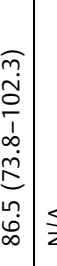 & \\
\hline 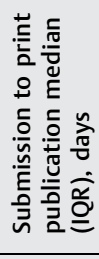 & 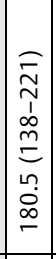 & 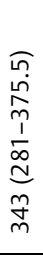 & 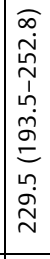 & 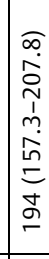 & $\frac{\pi}{z}$ & 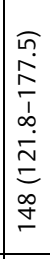 & 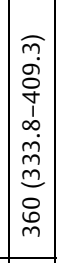 & 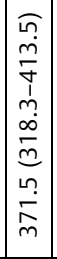 & 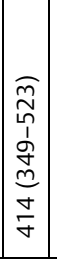 & 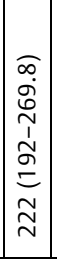 & 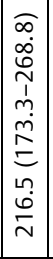 & 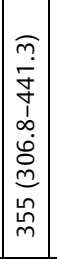 & $\frac{\pi}{z}$ & 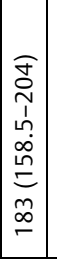 & $\frac{\pi}{z}$ & 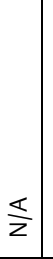 & $\frac{\ll}{z}$ & 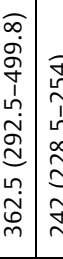 & 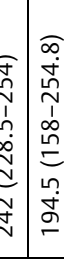 & 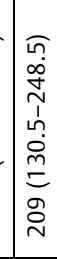 & $\mid \frac{5}{2}$ & 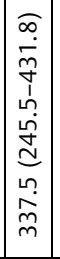 & & $\frac{5}{z}$ & 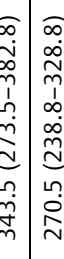 & 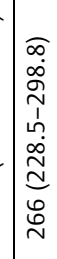 & 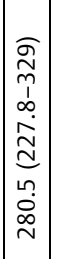 & 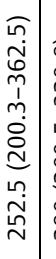 & 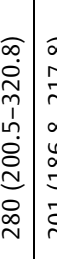 & \\
\hline 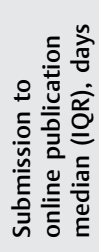 & 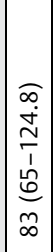 & 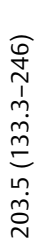 & 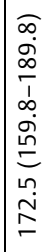 & $\mid \begin{array}{l}\widehat{\alpha} \\
\tilde{\tilde{m}} \\
\underline{1} \\
\underline{a}\end{array}$ & $\frac{\mathbb{s}}{z}$ & 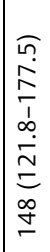 & 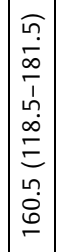 & 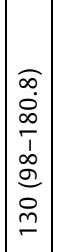 & 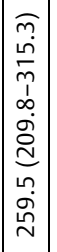 & 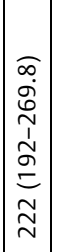 & 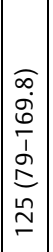 & 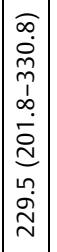 & 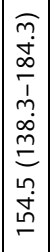 & 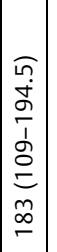 & $\frac{\pi}{z}$ & 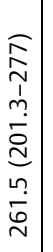 & $\frac{s}{z}$ & 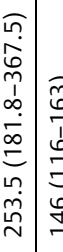 & 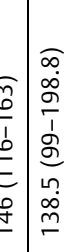 & $\frac{\varangle}{z}$ & 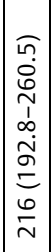 & 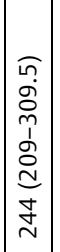 & 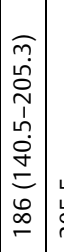 & 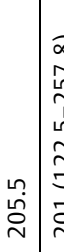 & 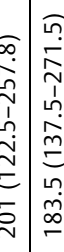 & 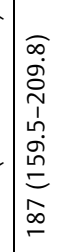 & 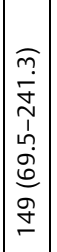 & 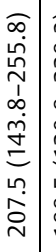 & 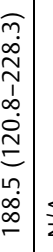 & \\
\hline 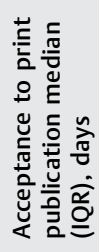 & 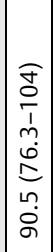 & 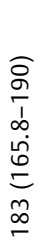 & 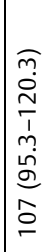 & & & & 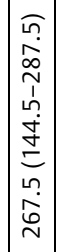 & 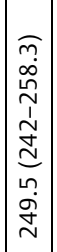 & 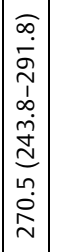 & 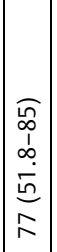 & $\begin{array}{l}0 \\
0 \\
1 \\
1 \\
\infty \\
\infty\end{array}$ & 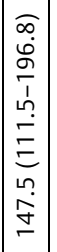 & $\frac{\pi}{z}$ & 过 & $\frac{\ll}{z}$ & & $\begin{array}{l}\dot{D} \\
0 \\
0 \\
1 \\
m \\
0 \\
0 \\
0 \\
0\end{array}$ & 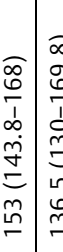 & 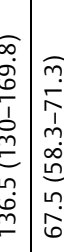 & 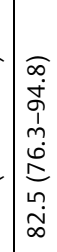 & 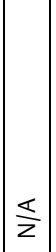 & 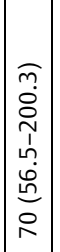 & & 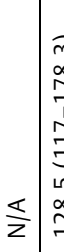 & 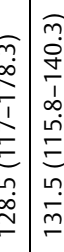 & $\begin{array}{l}\overline{5} \\
1 \\
1 \\
0 \\
0 \\
0 \\
0 \\
\text { n. }\end{array}$ & 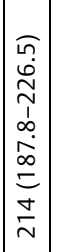 & 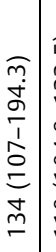 & & \\
\hline
\end{tabular}

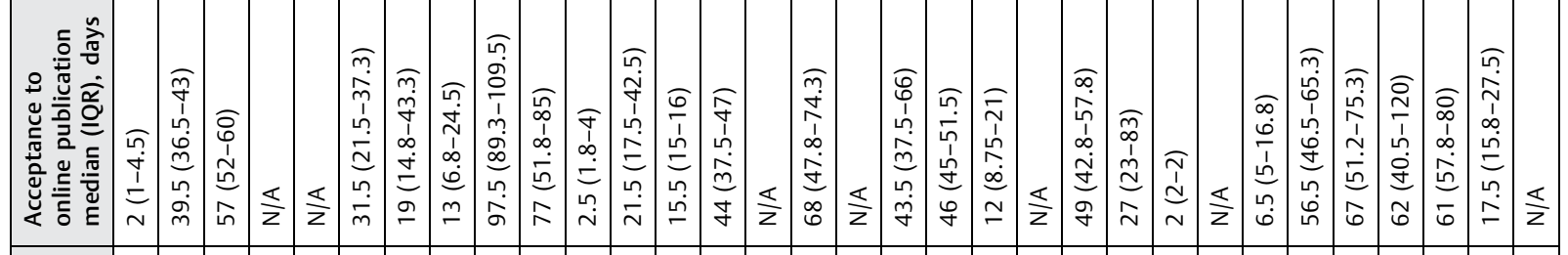

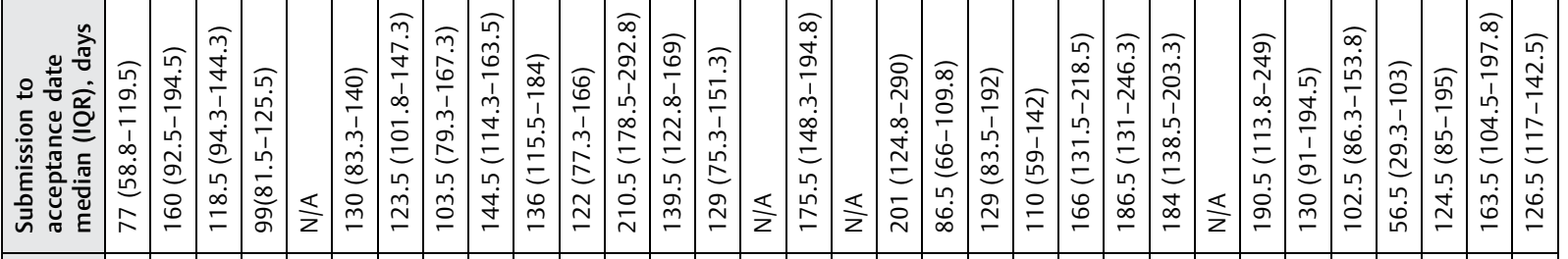

\begin{tabular}{|c|c|c|c|c|c|c|c|c|c|c|c|c|c|c|c|c|c|c|c|c|c|c|c|c|c|c|}
\hline ڤ̆ & 峁 & 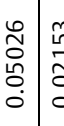 & 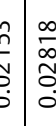 & 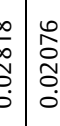 & $\begin{array}{l}\infty \\
0 \\
0 \\
0 \\
0\end{array}$ & $\mid \begin{array}{l}\bar{m} \\
\stackrel{n}{\underline{0}} \\
\dot{0}\end{array}$ & $\begin{array}{l}n \\
\hat{y} \\
0 \\
0 \\
0\end{array}$ & $\mid \begin{array}{l}0 \\
\overline{0} \\
0 \\
0\end{array}$ & 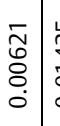 & 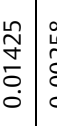 & 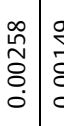 & 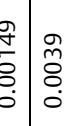 & 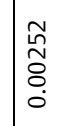 & $\begin{array}{c}\stackrel{0}{1} \\
\tilde{o} \\
\tilde{o} \\
0 \\
0\end{array}$ & 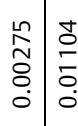 & 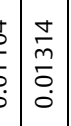 & 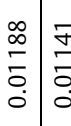 & 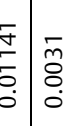 & $\left|\begin{array}{l}2 \\
0 \\
0 \\
0 \\
0 \\
0\end{array}\right|$ & 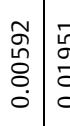 & 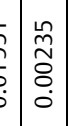 & $\begin{array}{c}\infty \\
\stackrel{0}{0} \\
\tilde{O} \\
0 \\
0\end{array}$ & & & $\begin{array}{l}0 \\
\tilde{\mathscr{o}} \\
0 \\
0\end{array}$ & \\
\hline & $\approx$ & 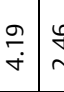 & 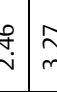 & 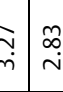 & $\underset{m}{\bar{N}}$ & $\mid \begin{array}{l}\infty \\
m \\
m\end{array}$ & $\stackrel{\infty}{\stackrel{\infty}{f} .}$ & $\stackrel{\underset{N}{N}}{\sim}$ & \begin{tabular}{c|c}
$\stackrel{\mathfrak{m}}{\mathrm{i}}$ & $\vdots$
\end{tabular} & 8 & 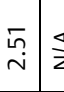 & $\frac{\Delta}{z} \mid \underset{i}{\sim}$ & $\cong$ & $\underset{\mathfrak{u}}{\stackrel{\sim}{i}}$ & 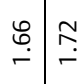 & 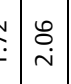 & 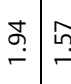 & مُ & $\underset{\sim}{\mathbf{J}}$ & $\stackrel{\sim}{\sim}$ & 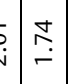 & $\stackrel{m}{r}$ & 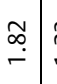 & 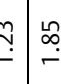 & $\stackrel{\infty}{\stackrel{\infty}{r}}$ & \\
\hline 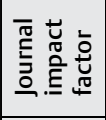 & $\begin{array}{l}\circ \\
\sigma \\
\end{array}$ & 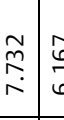 & & 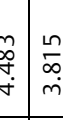 & $\begin{array}{l}\tilde{N} \\
\infty \\
m\end{array}$ & $\begin{array}{l}\frac{n}{6} \\
\dot{m} \\
\dot{m}\end{array}$ & $\bar{\gamma}$ & $\begin{array}{l}\stackrel{n}{n} \\
\stackrel{m}{m} \\
\end{array}$ & $m$ & $\begin{array}{l}\infty \\
\text { ô. } \\
\text {. }\end{array}$ & 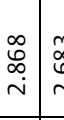 & 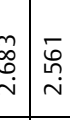 & 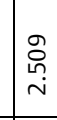 & 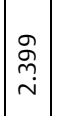 & 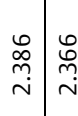 & 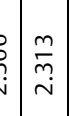 & 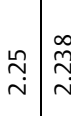 & 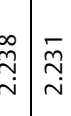 & $\mid \begin{array}{c}\infty \\
\stackrel{\sim}{\sim} \\
\dot{N}\end{array}$ & 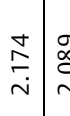 & 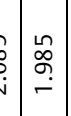 & 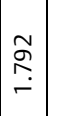 & & 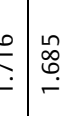 & $\begin{array}{l}3 \\
:\end{array}$ & \\
\hline & $\stackrel{5}{=}$ & 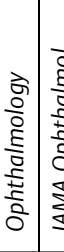 & 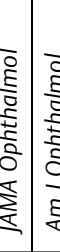 & 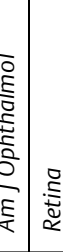 & 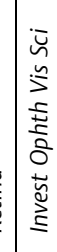 & 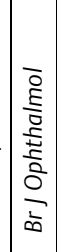 & 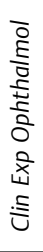 & 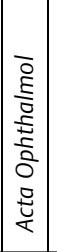 & 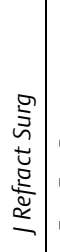 & 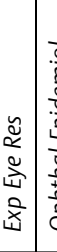 & 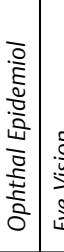 & 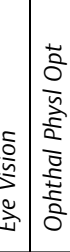 & 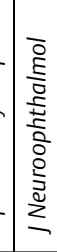 & 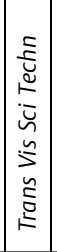 & 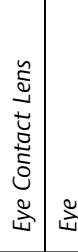 & 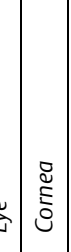 & 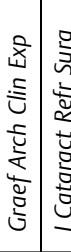 & 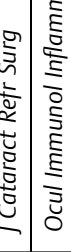 & 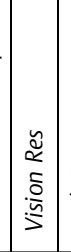 & 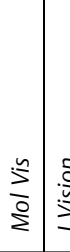 & 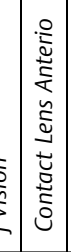 & 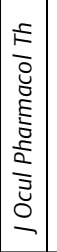 & 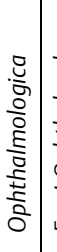 & 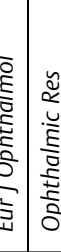 & 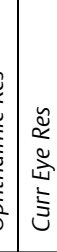 & \\
\hline
\end{tabular}




\begin{tabular}{|c|c|c|c|c|c|c|c|c|c|c|c|c|c|c|c|c|c|c|c|c|c|c|c|}
\hline 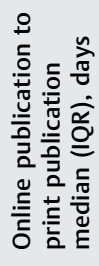 & 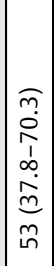 & 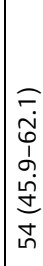 & $\frac{\mathbb{a}}{z}$ & $\frac{\pi}{z}$ & 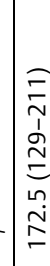 & 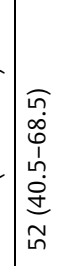 & 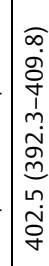 & 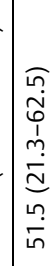 & $\frac{\pi}{z}$ & 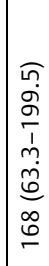 & $\frac{\varangle}{z}$ & 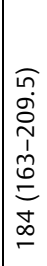 & 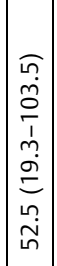 & $\mid \frac{\pi}{z}$ & $\frac{\mathrm{a}}{\mathrm{z}}$ & 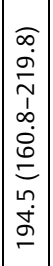 & 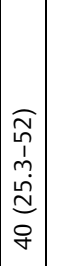 & 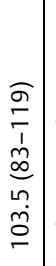 & 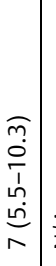 & $\begin{array}{r}\leq \\
z \\
z\end{array}$ & 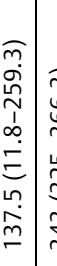 & $\begin{array}{l}c \\
m \\
z \\
m \\
m\end{array}$ & \\
\hline 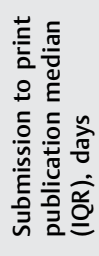 & 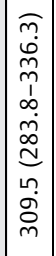 & 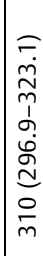 & | & 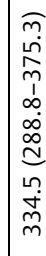 & 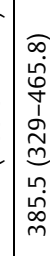 & 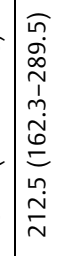 & $\begin{array}{l}\widehat{\partial} \\
0 \\
0 \\
1 \\
0 \\
0 \\
0 \\
0 \\
0 \\
i\end{array}$ & 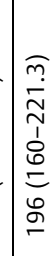 & $\frac{\varangle}{z}$ & 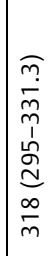 & $\frac{\alpha}{z}$ & 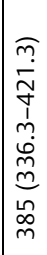 & 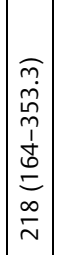 & 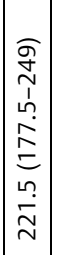 & $\frac{\pi}{z}$ & 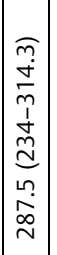 & 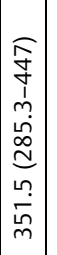 & 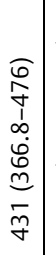 & 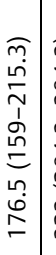 & 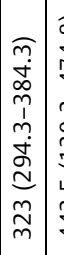 & 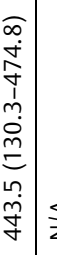 & $\begin{array}{l}\frac{1}{2} \\
\end{array}$ & \\
\hline 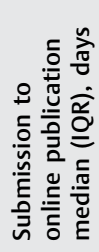 & 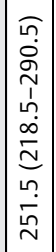 & 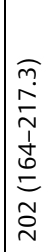 & 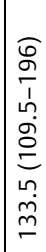 & $\frac{\pi}{z}$ & 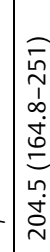 & 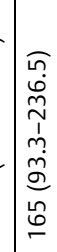 & 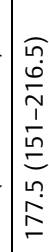 & 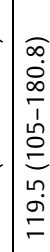 & 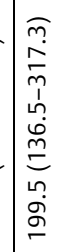 & 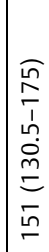 & 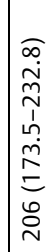 & 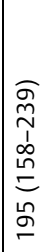 & 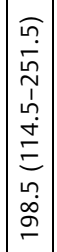 & $\frac{\pi}{z}$ & & 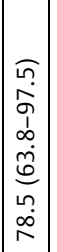 & 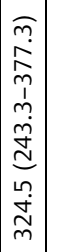 & 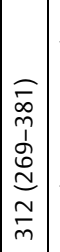 & 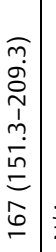 & $\frac{\varangle}{z}$ & 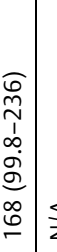 & & \\
\hline 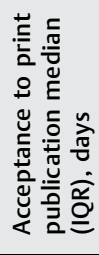 & 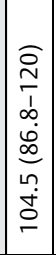 & $\frac{s}{z}$ & $\frac{\pi}{z}$ & 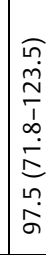 & 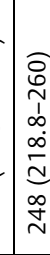 & 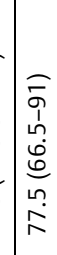 & 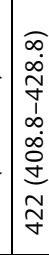 & 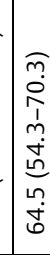 & $\frac{s}{z}$ & $\frac{\pi}{z}$ & $\frac{\Sigma}{z}$ & 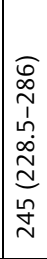 & 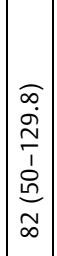 & 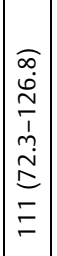 & 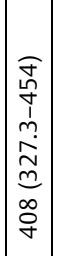 & 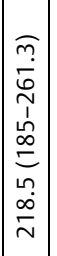 & 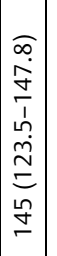 & 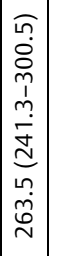 & 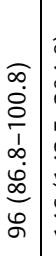 & 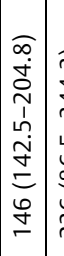 & 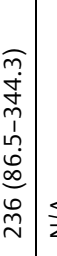 & & ) \\
\hline 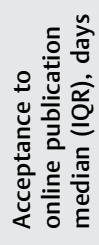 & 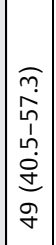 & 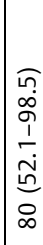 & 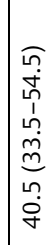 & $\mid \ll$ & 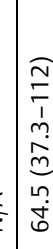 & 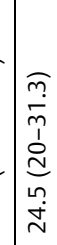 & $\begin{array}{c}\tilde{m} \\
\grave{i} \\
\vdots \\
0 \\
\dot{\theta} \\
m \\
\tilde{r}\end{array}$ & 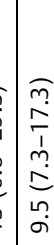 & 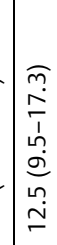 & $\frac{\varangle}{z}$ & $\mid \frac{\pi}{z}$ & 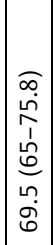 & 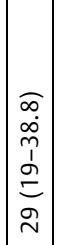 & $\frac{\pi}{z}$ & $\frac{\pi}{2}$ & 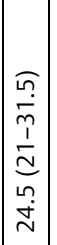 & 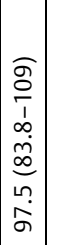 & 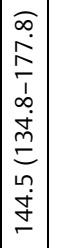 & 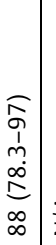 & $\frac{\pi}{z}$ & 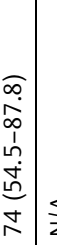 & & \\
\hline 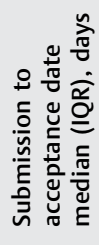 & 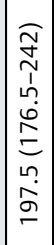 & 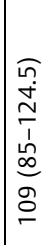 & 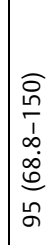 & 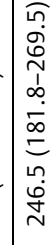 & 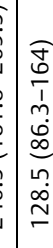 & 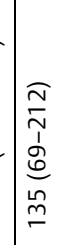 & 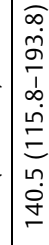 & 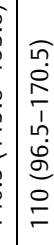 & 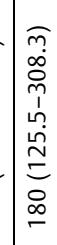 & $\frac{\pi}{z}$ & $\frac{\mathbb{s}}{z}$ & 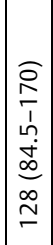 & 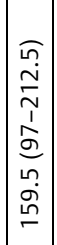 & 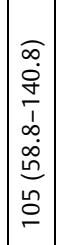 & & 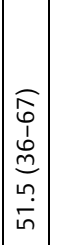 & 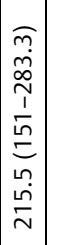 & 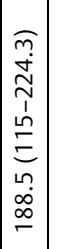 & 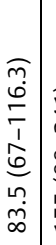 & 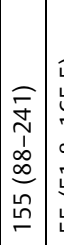 & 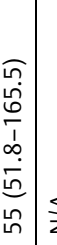 & $\frac{k}{z}$ & \\
\hline 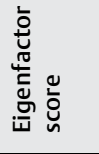 & $\mid \begin{array}{l}\vec{J} \\
\vec{\sim} \\
\tilde{O} \\
\dot{0}\end{array}$ & $\begin{array}{l}\overline{5} \\
\overline{8} \\
0 \\
0\end{array}$ & 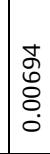 & $\begin{array}{l}\frac{m}{\hat{0}} \\
\stackrel{0}{0} \\
0\end{array}$ & 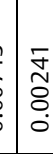 & 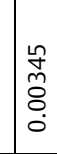 & 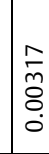 & $\mid \begin{array}{l}\infty \\
\bar{\delta} \\
\bar{\delta} \\
0\end{array}$ & 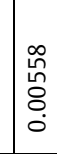 & $\begin{array}{l}\text { po } \\
\text { ○े } \\
0 \\
0\end{array}$ & 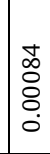 & 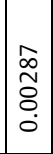 & \begin{tabular}{|l|} 
\\
$m$ \\
$\dot{m}$ \\
$\dot{0}$ \\
$\dot{0}$
\end{tabular} & 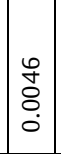 & 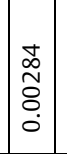 & $\begin{array}{l}0 \\
\stackrel{0}{0} \\
0 \\
0 \\
0\end{array}$ & $\mid \begin{array}{l}2 \\
\infty \\
\tilde{O} \\
0 \\
0 \\
0\end{array}$ & $\begin{array}{l}\bar{\sigma} \\
\bar{\sigma} \\
\dot{0} \\
\end{array}$ & $\begin{array}{l}0 \\
0 \\
\tilde{0} \\
0 \\
0 \\
0\end{array}$ & 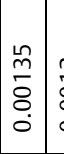 & 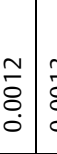 & & \\
\hline ڤِ & 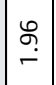 & $\frac{s}{z}$ & $\stackrel{\infty}{\stackrel{2}{=}}$ & 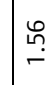 & $\stackrel{\oplus}{\stackrel{m}{m}}$ & $\stackrel{\stackrel{m}{m}}{\longrightarrow}$ & $\stackrel{\infty}{\check{r}}$ & 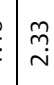 & 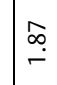 & $\stackrel{\stackrel{m}{r}}{=}$ & $\underset{\Sigma}{\stackrel{N}{-}}$ & $\begin{array}{l}\Omega \\
\hat{0} \\
0\end{array}$ & $\begin{array}{l}\hat{a} \\
0\end{array}$ & $\stackrel{\infty}{\stackrel{\infty}{\leftarrow}}$ & $\begin{array}{c}n \\
\stackrel{n}{0} \\
\dot{0}\end{array}$ & $\frac{s}{z}$ & $\mid \begin{array}{r}o \\
\dot{-}\end{array}$ & $\begin{array}{l}\bar{\sigma} \\
0 \\
0\end{array}$ & $\begin{array}{c}- \\
\infty \\
0 \\
\dot{0}\end{array}$ & 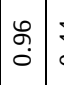 & \begin{tabular}{l|l}
$\stackrel{5}{*}$ \\
$\dot{0}$
\end{tabular} & ñ & D. \\
\hline 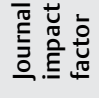 & $\mid \begin{array}{l}\hat{\leftrightarrow} \\
\stackrel{\leftrightarrow}{-} \\
\mid\end{array}$ & 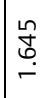 & ڤn? & $\stackrel{t}{\stackrel{t}{r}}$ & 蛊 & 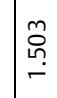 & 曽. & $\stackrel{?}{\stackrel{0}{.}}$ & 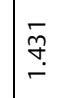 & 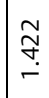 & 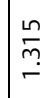 & $\left|\begin{array}{l}n \\
m \\
m \\
\sim\end{array}\right|$ & $\mid \begin{array}{l}\stackrel{\sim}{0} \\
\stackrel{1}{\sim} \\
\mid\end{array}$ & $\mid \begin{array}{c}\stackrel{\infty}{\infty} \\
\stackrel{+}{\leftarrow}\end{array}$ & $\stackrel{\stackrel{m}{m}}{\stackrel{m}{-}}$ & $\left|\begin{array}{c}\hat{\jmath} \\
\hat{\sigma} \\
\dot{-}\end{array}\right|$ & $\mid \begin{array}{l}\stackrel{0}{h} \\
\stackrel{\leftrightarrow}{0}\end{array}$ & $\begin{array}{l}\stackrel{u}{\leftrightarrow} \\
\stackrel{-}{*}\end{array}$ & $\begin{array}{l}\hat{\sigma} \\
\text { o. } \\
0\end{array}$ & 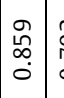 & 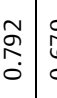 & & है \\
\hline 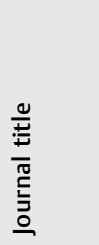 & 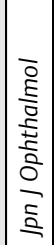 & 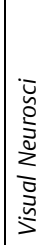 & 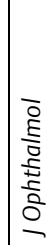 & 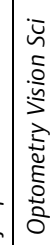 & 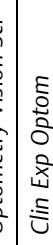 & 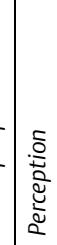 & 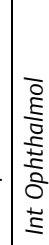 & 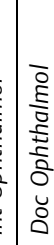 & 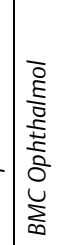 & 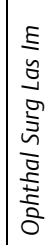 & 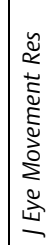 & 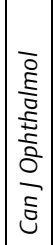 & 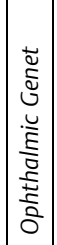 & 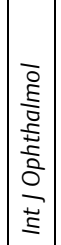 & 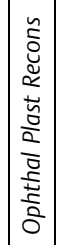 & 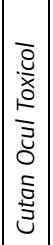 & $\mid$ & 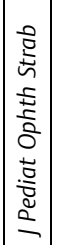 & 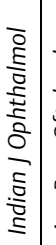 & 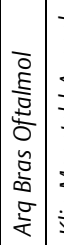 & 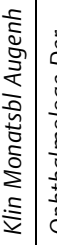 & & \\
\hline
\end{tabular}



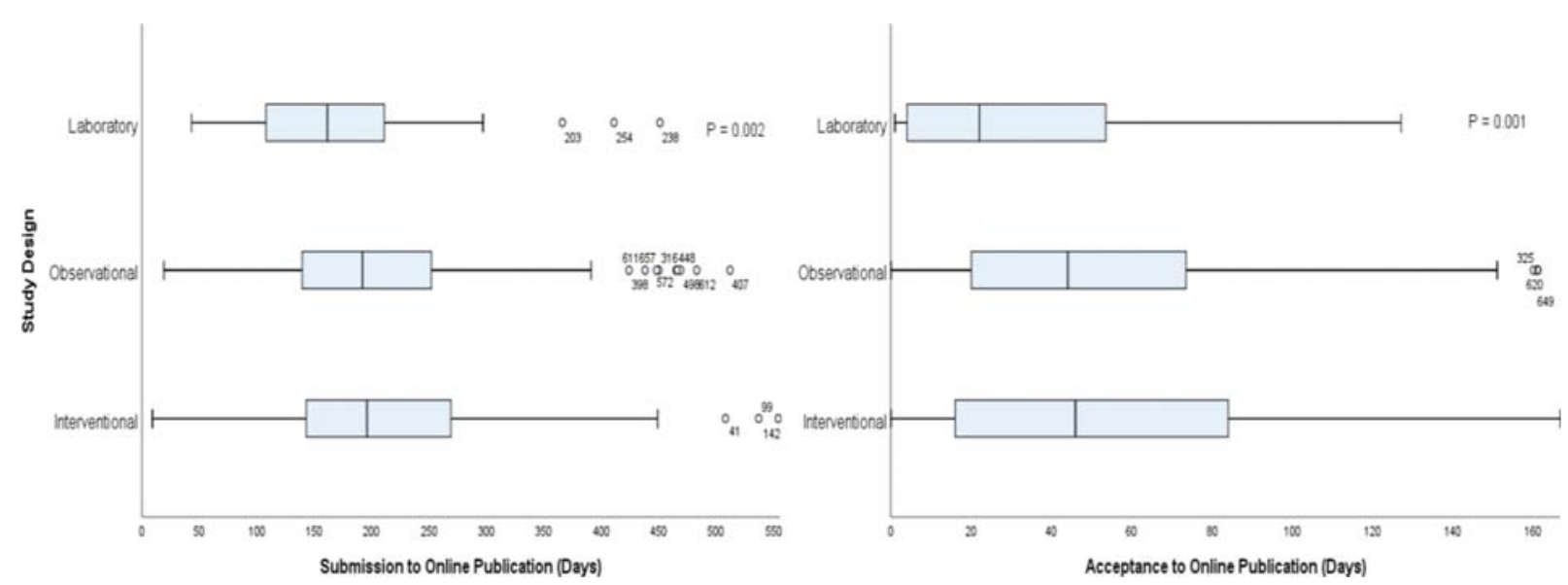

Fig. 1 Box and Whisker plots of time lag differences between laboratory, observational, and interventional study designs. (A) Depicts differences in time lag from submission to online publication and (B) depicts acceptance to online publication. Kruskal-Wallis Test showed a statistically significant difference in both parameters of $p=0.002$ and $p=0.001$, respectively. Post hoc analysis with Mann-Whitney $U$-test found laboratory experiments had a statistically significant difference when compared with both groups and no differences between observational and interventional studies. Outliers are represented with circles.

selected articles, 541 articles were clinical, 110 were basic science papers, and 6 articles were not assigned to either category due to their theoretical/mathematical nature. Fortyone study results were negative (null hypothesis was not rejected) and 610 were positive. For study design, 185 articles were interventional, 362 were observational, and 110 were laboratory experiments.

\section{Impact of Study Design on Speed to Publication}

Laboratory experiments differed from both observational and interventional studies in time lag from submission to online publication $(p=0.002)$ and from acceptance to online publication $(p<0.001)$, but there was no difference between observational and interventional studies (-Fig. 1). Laboratory experiments were published approximately 30 to 36 days faster than other study designs, with a median time of 161.5 days from submission date to online publication date compared with 190 days in observational studies $(p<0.001)$ and 196 days in interventional studies $(p<0.001)$. After articles were accepted, laboratory experiments were published online twice as fast as observational and interventional studies with a median time of 22 days compared with 44 days for observational $(p=0.002)$ and 46 days for interventional studies $(p=0.002)$. No other statistically significant difference existed between study designs for time from acceptance to print publication $(p=0.341)$, submission to print publication $(p=0.07)$, submission to acceptance $(p=0.115)$, submission to revision $(p=0.617)$, revision to acceptance $(p=0.608)$, nor online publication to print publication ( $p=0.267$ ).

\section{Publication Timing Characteristics}

The median time lag for all journals from submission to online publication was 187 days with an IQR of 150 to 211.75 days. The median time lag from submission to print publication was 284 days with an IQR of 215 to 352 days. The median days from online publication to print publication was 84.75 days with an IQR of 52.3 to 163.5 days. The difference in the time from submission to online publication and submission to print publication for all journals was statistically significant $(p<0.001)$. The median times from submission to acceptance and from acceptance to online publication were 129 days (IQR: 105 to 166 ) and 43.5 days (IQR: 16.5-67), respectively. The median time from acceptance to print publication was 134 days with an IQR of 86.5 to 227.25 days. The median times from submission to revision and revision to acceptance were 103.3 days (IQR: 83.9-147.1) and 12 days (IQR: $6.5-16.25)$, respectively.

\section{Correlations of Publication Speed with Journal Impact Factor, CiteScore, Eigenfactor Score}

All three examined journal measures significantly positively correlated with each other: JIF to CS had an $r$ value of 0.815 $(p<0.001)$, JIF to ES had an $r$ of $0.667(p<0.001)$, and CS to ES had an $r$ value of $0.618(p<0.001)$. A statistically significant correlation was found between the JIF and the time from acceptance to online publication $(r=-0.332, p=0.034)$. A statistically significant correlation was found between the CS and three parameters: submission to print publication $(r=-$ $0.326, p=0.04)$, acceptance to print publication $(r=-0.375$, $p=0.017)$, and acceptance to online publication $(r=-0.447$, $p=0.005)$. Neither statistically significant correlations were found for ES nor any other parameters with CS and JIF (-Fig. 2).

\section{Comparison to 2010 Study}

Comparative analysis to Chen et al revealed no statistically significant difference in the changes from 2010 to 2018 for the three parameters measured in their study. The time lag from acceptance to print in 2010 was 87 days (IQR: 58.5 to 166.8 ) for all journals compared with 142.5 days (IQR: 96 to $218.5)$ in $2018(p=0.113)$. Time from submission to acceptance and submission to print was 133.5 days in 2010 and 128.5 days in 2018 from submission to acceptance $(p=0.635)$, and 244 days in 2010 to 284 days in 2018 from submission to print publication $(p=0.215)$. Similar to Chen 
A

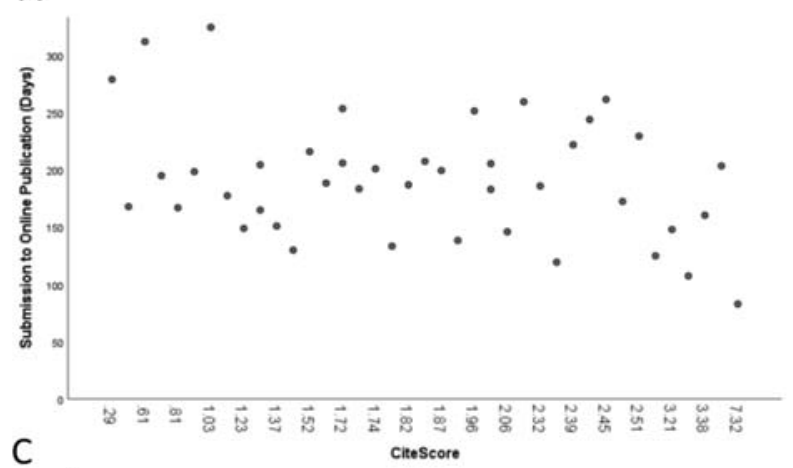

B
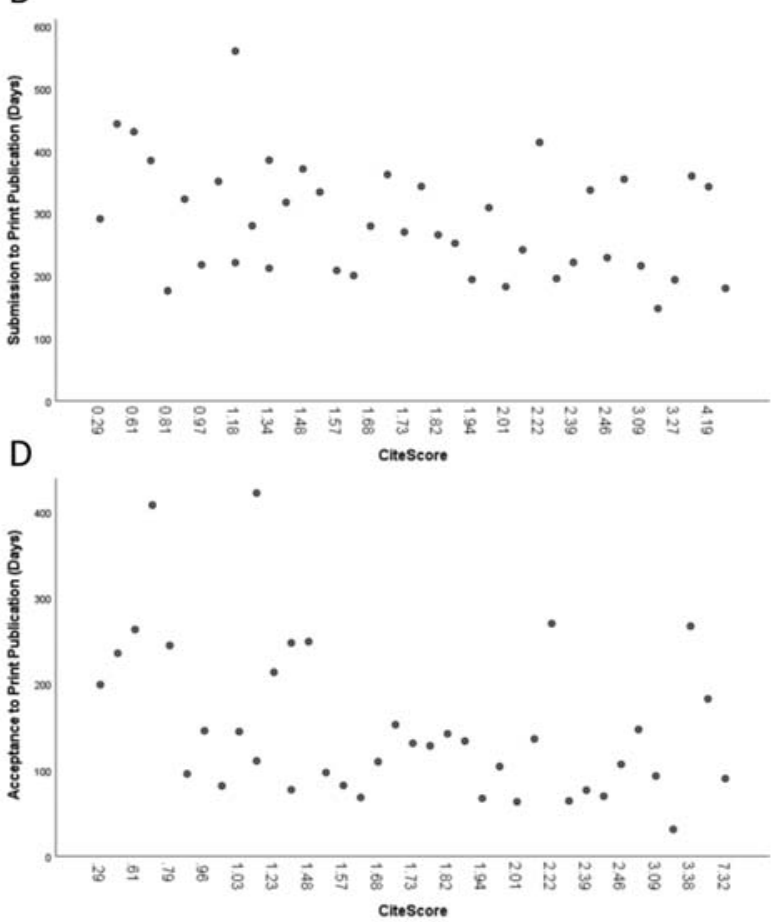

Fig. 2 Scatterplots demonstrating the correlations between CiteScore and various time periods. Spearman's Correlation of CiteScore with (A) time from submission to online publication is $r=-0.224(p=0.154),(B)$ time from submission to print publication is $r=-0.326(p=0.04),(C)$ time from acceptance to online publication is $r=-0.447(p=0.005)$, and $(\mathrm{D})$ time from acceptance to print publication is $r=-0.375(p=0.017)$.

et al, our study found no correlation between JIF and days from acceptance to print publication, submission to print publication, and submission to acceptance date $(p=0.245$, 0.167 , and 0.768 , respectively).

\section{Discussion}

As technology and innovation continue to accelerate in ophthalmology, faster times to publication are necessary to keep the rate of knowledge dissemination on par with the rate of knowledge acquisition. Some argue, however, that faster publication times may be detrimental, citing a lower quality peer review process as a possible repercussion of seeking faster publication times. ${ }^{9,10}$ This debate has become more prominent during the COVID-19 viral pandemic. Major medical journals, including the New England Journal of Medicine and Lancet, have issued retractions for COVID-19related articles that were fast tracked to publication and whose results influenced trial design by major institutions, including the World Health Organization. ${ }^{11}$ Relevant to ophthalmology, an expedited article in Lancet characterizing optical coherence tomography (OCT) findings in patients with COVID-19 infection has received criticism for misinterpretation of normal retinal anatomy in the images, indicating that perhaps the review process was not thorough enough. ${ }^{12,13}$

Finding the perfect balance between being timely and still ensuring accuracy is critical for any peer-reviewed journal. Our results suggest that this pressure is greatest on the most prominent journals in ophthalmology, as online publication speed was significantly and positively correlated with higher bibliometric measures of journal's "impact" and "prestige." As their higher status of journal impact and prestige demonstrate that they are the most consumed information in the field, these journals may feel the need to publish data as rapidly as possible when new changes in the field arise. This can be seen with the current viral pandemic in which most articles involving COVID-19 were submitted to and published in these journals. Unfortunately, likely due to attempts to disseminate this information immediately, key data were overlooked and articles were ultimately retracted. This expectation to publish articles as soon as possible for the public may have been the reason for the fast turnaround time of publication and consequently, the errors made. Alternatively, it is possible that these journals may have more resources available and allows them to expedite publication times for submissions faster than other less regarded journals. This may also explain the correlation to faster online publication speeds found in this study or can point to possibly a combination of both the need to publish quickly and having more access to resources as reasons. More research in this field of study is required to clearly delineate this correlation.

It is noteworthy that JIF was not correlated to submission to print, acceptance to print, or submission to acceptance times. This implies that the greatest portion of acceleration by "higher impact" journals occurs when taking an article from acceptance to online publication. Indeed, the time from submission to online publication was significantly shorter than time to print publication, allowing editors more time to proof articles prior to commitment to physically printing. 
Still, once available online, peer-reviewed manuscripts are considered vetted by the publishing journal and consumed by the scientific public regardless of the pending print status. $^{14-16}$ Thus, we would argue that ophthalmology's highly regarded journals must be cautious and should approach time from submission to online publication as the most critical "rate-limiting step" where errors and research gaps must be caught.

One would expect that because of the prolonged time and effort that it takes to carry out laboratory experiments, especially additional validation experiments requested after peer review, the time to publish laboratory experiments would be longer than clinical studies. In fact, the opposite was found to be true. There are various possible explanations. One study reported that the "impact" of clinical studies may be deemed to be smaller than that of basic science studies in the literature. ${ }^{17}$ This perception of higher impact of basic science articles may be the cause of shortened publication times; laboratory studies submitted to and published in ophthalmology journals, which tend to be more clinically oriented, may be viewed as important findings that would benefit clinicians instantly or encourage immediate pursuit of applied research in these areas. Laboratory studies are also not as commonly published in ophthalmology journals, possibly inflating the perceived importance in the instances that these articles are submitted and published, leading to faster publication times. Similarly, a shift toward widespread interest in translational medicine in recent years may be a contributing factor to necessitating faster publication times of the basic sciences. ${ }^{18}$ Research on rates of basic science submission and the immediate impact of basic science in the clinical setting is scarce and future research investigating these matters is warranted.

While the JIF was not initially intended to be used as a method for researchers to rank the top journals in a field of study, over the years, it has become the gold standard for this purpose. ${ }^{19}$ Likewise, other bibliometric measures to be evaluated for this purpose have gained traction. Although each measure accounts for different characteristics in a journal, as seen here for ophthalmology journals and in the literature for other fields of study, these factors are strongly positively correlated in relation to each other. ${ }^{20}$ For this reason, one suggested method for evaluating a journal's "prestige" is to evaluate and rate various bibliometric measures simultaneously and rank the journals in a given field based on highest ratings across these measures. ${ }^{5}$ This would counter some of the inherent flaws of each bibliometric measure and allow for a more robust evaluation of top journals in a field.

\section{Limitations}

There were several limitations to this study. The bibliometric measures assessed are only three of countless available measures. The use of three measurements arose from recent recommendations that suggest relying less on only JIF, and instead including several bibliometric measures for assessment of a journal. ${ }^{21-23}$ Due to random selection, this study did not achieve a high-enough proportion of basic science and negative result studies to determine whether those parameters impact publication times. Future studies may aim to actively search for these articles during data collection to have a more representative sampling for basic science studies and negative studies. Another limitation was the absence of revision dates and counts in the majority of journals. Time to revision from article to article likely varies widely due to several factors and is valuable information that is not readily available. For this reason, we did not continue with our assessment of revision times, although we recorded these values whenever available. Future studies addressing these variables would likely provide more definitive answers to the role these possible confounders may play. Lastly, this study was limited to only ophthalmology journals. One would suspect that the speed at which articles are published likely plays a role in every field of study, and that repeating this study in other fields may provide invaluable information for these specialties. Additionally, there are ophthalmic/eye research journal articles that are published in nonophthalmology journals and we did not assess those articles.

\section{Conclusion}

In conclusion, our study demonstrates that ophthalmology journals with higher bibliometric measure scores have significantly higher online publication speed, emphasizing the importance placed on rapid dissemination of knowledge by these most widely read journals. Publishing manuscripts faster must be balanced with the risks of retractions, corrections, and other errors that may have major downstream effects given the potential impact of these journals' articles on clinical care and ongoing scientific research. ${ }^{24,25}$ Future studies may shed light on how common these speed-influenced errors are and may help determine the optimal publication speed to balance the simultaneous objectives of scientific accuracy and expediency.

\section{Conflict of Interest}

J.S. reports personal fees from Alcon, personal fees from Regeneron, personal fees from Oxurion, outside the submitted work. A.E.K. reports personal fees from Allergan, personal fees from Alimera Sciences, personal fees from Bausch Health, grants and personal fees from Genentech/ Roche, personal fees from Regeneron, grants from Second Sight, outside the submitted work. There are no other conflicts of interest from the remaining authors.

\section{Acknowledgments}

Financial support: the Bascom Palmer Eye Institute received funding from NIH Core Grant P30EY014801, Department of Defense grant no.: W81XWH-13-1-0048, and a Research to Prevent Blindness Unrestricted Grant. The sponsors and funding organizations had no role in the design or conduct of this research.

Access to data: two authors, J.S. and R.N, had full access to all the data in the study and take responsibility for the integrity of the data and the accuracy of the data analysis. 


\section{References}

1 Brink PA. Article visibility: journal impact factor and availability of full text in PubMed Central and open access. Cardiovasc J Afr 2013;24(08):295-296

2 Chen $\mathrm{H}$, Chen $\mathrm{CH}$, Jhanji V. Publication times, impact factors, and advance online publication in ophthalmology journals. Ophthalmology 2013;120(08):1697-1701

3 Roldan-Valadez E, Orbe-Arteaga U, Rios C. Eigenfactor score and alternative bibliometrics surpass the impact factor in a 2-years ahead annual-citation calculation: a linear mixed design model analysis of Radiology, Nuclear Medicine and Medical Imaging journals. Radiol Med (Torino) 2018;123(07):524-534

4 Roldan-Valadez E, Salazar-Ruiz SY, Ibarra-Contreras R, Rios C. Current concepts on bibliometrics: a brief review about impact factor, Eigenfactor score, CiteScore, SCImago journal rank, sourcenormalised impact per paper, h-index, and alternative metrics. Ir J Med Sci 2019;188(03):939-951

5 Franchignoni F, Özçakar L, Negrini S. Basic bibliometrics for dummies and others: an overview of some journal-level indicators in physical and rehabilitation medicine. Eur J Phys Rehabil Med 2018;54(05):792-796

6 Kianifar H, Sadeghi R, Zarifmahmoudi L. Comparison between impact factor, Eigenfactor metrics, and scimago journal rank indicator of pediatric neurology journals. Acta Inform Med 2014;22(02):103-106

7 Van Noorden R. Controversial impact factor gets a heavyweight rival. Nature 2016;540(7633):325-326

8 Kumar A, Cheeseman R, Durnian JM. Subspecialization of the ophthalmic literature: a review of the publishing trends of the top general, clinical ophthalmic journals. Ophthalmology 2011;118 (06):1211-1214

9 Schachat AP, Bartley GB. Faster is better. Ophthalmology 2013; 120(08):1513-1514

10 Morris ZS, Wooding S, Grant J. The answer is 17 years, what is the question: understanding time lags in translational research. J R Soc Med 2011;104(12):510-520

11 Redden E. Rush to publish risks undermining COVID-19 research. Available at: https://www.insidehighered.com/news/2020/06/ 08/fast-pace-scientific-publishing-covid-comes-problems. Accessed September 30, 2020
12 Marinho PM, Marcos AAA, Romano AC, Nascimento H, Belfort R Jr. Retinal findings in patients with COVID-19. Lancet 2020;395 (10237): 1610

13 Vavvas DG, Sarraf D, Sadda SR, et al. Concerns about the interpretation of OCT and fundus findings in COVID-19 patients in recent Lancet publication. Eye (Lond) 2020

14 Ollé C, Borrego Á. A qualitative study of the impact of electronic journals on scholarly information behavior. Libr Inf Sci Res 2010; 32(03):221-228

15 Bauchner $\mathrm{H}$. The rush to publication: an editorial and scientific mistake. JAMA 2017;318(12):1109-1110

16 Rawlinson C, Bloom T. New preprint server for medical research. BMJ 2019;365:12301

17 van Eck NJ, Waltman L, van Raan AF, Klautz RJ, Peul WC. Citation analysis may severely underestimate the impact of clinical research as compared to basic research. PLoS One 2013;8(04): e62395

18 Smith C, Baveja R, Grieb T, Mashour GA. Toward a science of translational science. J Clin Transl Sci 2017;1(04):253-255

19 Archambault É, Larivière V. History of the journal impact factor: contingencies and consequences. Scientometrics 2009;79(03): 635-649

20 Okagbue HI, Teixeira da Silva JA. Correlation between the CiteScore and Journal Impact Factor of top-ranked library and information science journals. Scientometrics 2020;124(01): 797-801

21 Nestor MS, Fischer D, Arnold D, Berman B, Del Rosso JQ. Rethinking the journal impact factor and publishing in the digital age. J Clin Aesthet Dermatol 2020;13(01):12-17

22 Fersht $A$. The most influential journals: impact factor and Eigenfactor. Proc Natl Acad Sci U S A 2009;106(17):6883-6884

23 Casadevall A, Fang FC. Causes for the persistence of impact factor mania. MBio 2014;5(02):e00064-e14

24 Abdollahi M, Gasparyan AY, Saeidnia S. The urge to publish more and its consequences. Daru 2014;22(01):53

25 Guraya SY, Norman RI, Khoshhal KI, Guraya SS, Forgione A. Publish or Perish mantra in the medical field: A systematic review of the reasons, consequences and remedies. Pak J Med Sci 2016;32 (06):1562-1567 